\title{
FACIAL EXPRESSIONS INFLUENCE KIN RECOGNITION ACCURACY
}

\author{
Vanessa Fasolt, Iris J. Holzleitner, Anthony J. Lee, Kieran J. O’Shea, \\ Lisa M. DeBruine
}

Institute of Neuroscience and Psychology, University of Glasgow, UK

v.fasolt.1@research.gla.ac.uk

\begin{abstract}
Kinship informs the allocation of pro-social and sexual behaviour. In addition to the ability to detect kin who are directly related to the observer, humans are also able to detect relatedness among others who are not related to themselves based on facial cues of relatedness. However, it is unclear what exact facial cues inform these kinship judgments. Facial expression might be one candidate, as it has been shown that a computer kin-detection algorithm can match relatives accurately when the stimuli are smiling. The current study investigated whether a smiling facial expression increases the accuracy of judging relatedness compared to a neutral facial expression in human raters. The stimuli were images of 50 sibling pairs and 50 unrelated pairs (aged 3-17 years) matched for age, ethnicity and sex. The stimuli included both neutral and smiling versions of each individual. Raters $(N=77)$ were asked to judge whether the presented pairs were related or not in one of two counterbalanced versions of the study, where the same stimuli were never presented as both smiling and neutral to the same rater, and the expression within the pair was always the same. Binary relatedness judgments were analysed using binomial logistic mixed regression. Contrary to expectations, smiling decreased the accuracy of relatedness judgments compared to a neutral facial expression. When shown with a smiling expression compared to a neutral one, related pairs were judged to be related less often, while unrelated pairs were judged to be related more often. Evidence that the upper face is mostly used for kinship judgments suggests that smiles could distort or distract from other, more reliable cues of kinship.

Pre-registration, data and code available at https://osf.io/58ewu/.
\end{abstract}

Keywords: Kinship, Face Research, Facial Similarity, Allocentric Kin Recognition. 


\section{INTRODUCTION}

Humans, along with other animals, possess the ability to distinguish between kin and non-kin, which is integral to the development of social, sexual and parental behaviours (Chapais \& Berman, 2004; Hepper, 2005; Lieberman, Tooby, \& Cosmides, 2007). The ability to detect kin allows individuals to favour their relatives by displaying prosocial behaviour (Hamilton, 1964) and optimise their reproductive behaviour by avoiding inbreeding (Bateson, 1983). One cue used for kin recognition is visual processing of physical similarities, or phenotype matching (for a review, see Penn \& Frommen, 2010). Research shows that those who share facial similarities with the observer are favoured in social contexts (see DeBruine, Jones, Little, \& Perrett, 2008 for a review). For example, studies have shown that in economic games, raters displayed increased levels of cooperation and trust with players whose faces were more similar to their own (DeBruine, 2002; Krupp, DeBruine, \& Barclay, 2008). Similarly, experimentally increased facial resemblance results in increased intentions about investment in children (DeBruine, 2004; Platek et al., 2003), while perceptions of facial resemblance between siblings predict altruistic behaviours and emotional closeness (Lewis, 2011).

In addition to the ability to detect kin who are directly related to the observer, humans also demonstrate the capacity to detect relatedness among others who are not related to themselves. This ability is referred to as allocentric kin recognition and has been illustrated repeatedly in previous literature, for both parent-child pairs (Alvergne, Perreau, Mazur, Mueller, \& Raymond, 2014; Bressan \& Dal Martello, 2002; Bressan \& Grassi, 2004; Nesse, Silverman, \& Bortz, 1990) and sibling pairs (Dal Martello, DeBruine, \& Maloney, 2015; DeBruine et al., 2009; Maloney \& Dal Martello, 2006).

Limited research has looked into which facial cues are actually used when making kinship judgments. Dal Martello and Maloney (2006) conducted two experiments to determine where kin recognition signals are in the human face. In both experiments, observers were shown pairs of photographs of children's faces and asked to judge sibship. The first experiment measured performance when either the upper or lower half of the face was masked, and the second experiment measured performance when either the mouth or eye region was masked. They found that kin judgment accuracy deteriorated significantly when the upper half of the face was masked, but found no difference in kin recognition when the lower face was masked. This suggests that cues used for kinship judgments are situated primarily in the upper half of a child's face. These findings highlight that different areas of the face are of different importance when making judgements about relatedness.

Following research finding that the right and left cerebral hemispheres are differentially involved in the perception of facial emotions and face identities (Butler et al., 2005; Coolican, Eskes, McMullen, \& Lecky, 2008; Megreya \& Havard, 2011; Rhodes, 1985), lateralisation of the face as a kinship cue has also been considered. However, Dal Martello and Maloney (2010) concluded that neither the left side of the face or the right side was superior in revealing information of kinship and that symmetry cues were not utilised when evaluating kinship. Dal Martello, DeBruine \& Maloney (2015) also did not find an effect of inversion or rotation on kinship judgment accuracy, although face inversion disrupts other perceptions such as identity and expression. The results of the 
above studies suggest that the process of kinship detection is specialised and differs from the way in which other features of the face are processed.

Based on the above evidence, it is unclear what role facial cues of emotions might play in kinship judgments. Some evidence suggests that a smiling facial expression aids some facial judgments, as for example nationality is identified correctly more frequently when the stimuli's facial expression is smiling rather than neutral (Marsh, Elfenbein, \& Ambady, 2003, 2007). Consequently, it might be that facial expressions can be cues to kinship, or enhance kinship recognition. However, facial expressions might be processed differently from cues to kinship and hinder kinship recognition. One study somewhat addressed this question, using a computer verification task to assess kinship of short videos of faces showing a dynamic, spontaneous smile (Dibeklioglu, Ali Salah, \& Gevers, 2013). The computer verification task achieved a kinship detection accuracy of $73 \%$, which is slightly superior to human kinship detection rates. Most human kinship detection studies have used stimuli with neutral expressions, so it is unclear what effect a smiling facial expression would have on kinship detection accuracy. Moreover, the computer verification task did not compare its accuracy levels for smiling faces to accuracy levels for neutral faces, therefore very little can be said about whether a smiling facial expression influences kinship recognition at all.

Nevertheless, based on this successful computer verification task of smiling kin and Marsh, Elfenbein and Ambady's (2003, 2007) findings that smiles aid nationality identification, smiles might be a helpful cue to kinship also. Smiles might partially be a contextual cue of kinship, with smiles within a family being more similar than smiles of strangers.

In light of the above, the current investigation is the first to explore the effect of facial expression on human raters' ability to recognise kin. This will help to provide further information about which factors can influence allocentric kin recognition. We hypothesised that relatedness will have a main effect, whereas raters are more likely to judge related pairs as related, and that a smiling facial expression will increase the accuracy of this judgment compared to a neutral expression.

\section{METHODS}

The methods for this study were pre-registered on the Open Science Framework at https://osf.io/ujnfp/. The planned analysis script is available at this site, as well as details about the hypotheses, stimuli and procedure. All procedures below follow this pre-registration exactly. The final data and analysis including improved visualisations and additional analyses suggested by reviewers can be found at https://osf.io/ggc79/. Any not pre-registered analysis is pointed out clearly.

\section{Stimuli}

Stimuli were collected from children visiting a local science centre who volunteered to take part in a study of facial cues of family relatedness. Parental consent and child assent were obtained for each child to use their face photograph in studies of family 
resemblance detection. Children were photographed with a smiling expression and then a neutral expression looking straight at the camera with hair pulled back and any glasses, scarves, and hats removed. The specific procedures for image collection are available at https://osf.io/bvtnj.

From a set of approximately 1500 images of individuals of varying ages, sex and relatedness, we algorithmically chose the maximum number of sibling pairs fitting a number of criteria. Both siblings were required to be genetically related and non-twin full siblings under the age of 18 . We also required that an age-matched (within 1 year), ethnicity-matched, and sex-matched foil image was available from family units that were not represented elsewhere in the image set. Specifically, the two individuals in each sibling pair are related to each other, but not to any other individual in the set, while all individuals in unrelated pairs are related to no individuals in the set. We are not able to exclude the possibility that stimuli might be distantly related without our knowledge.

This procedure produced 50 sibling pairs and 50 matched unrelated pairs. In each group, 13 pairs were both male, 15 pairs were both female, and 22 pairs were male and female. The individuals ranged from 3 to 17 years of age (mean age $=9.44, \mathrm{SD}=2.92$ ) and the age difference between individuals in a pair ranged from 0 to 7 (mean $=2.96$, SD $=1.64)$ years, meaning that at least one pair was born within 12 months of each other without being twins.

\section{Procedure}

Recruitment of raters was done online through social media (e.g., Facebook) and social bookmarking sites. The study itself was completed online at faceresearch.org and lasted around 10 minutes.

Raters were randomly assigned to one of two counterbalanced versions of the study. Each rater was presented with 100 stimuli pairs, which were presented in a random order. Half of these stimuli pairs were shown smiling and half with a neutral expression, which ensured that raters rated both smiling and neutral faces. Raters were, however, never shown the same pair with both expressions, as the pairs that were shown smiling in one version of the study were shown neutral in the other version. Before the study began, raters received the following instructions: "In this experiment you will be shown 100 pairs of faces. Some are siblings, some are an unrelated pair. You will be asked to determine whether each pair is unrelated or related." They were shown one pair of child faces at a time and chose their answer by clicking on buttons labelled "unrelated" or "related" without any time restrictions. We do not know whether any of the raters were familiar with any of the individuals shown during the study, however, recruitment for data collection and recruitment for the online study were done separately and on separate platforms. Photographs were mainly taken of local families in the local science centre, while raters from all over the world took part in the online study, making it unlikely, but not impossible, that they would know a small number of the individuals shown.

\section{Raters}

The kinship task was started by 81 people; we excluded 4 raters who did not rate all 100 stimuli, and were therefore left with 77 raters for analyses. After the exclusions, the 
distribution of raters looked as follows: 40 raters completed version A of the study and 37 raters completed version B of the study.

The responses from the two versions were analysed together to be able to compare the performance of the raters within pairs of stimuli. Overall, the responses from 28 men (mean age $=26.89 ; \mathrm{SD}=12.5)$ and 49 women $($ mean age $=26.15 ; \mathrm{SD}=11.27)$ were analysed.

\section{RESULTS}

We used a logistic mixed model to predict relatedness judgments from actual relatedness (effect coded as related $=+0.5$ and unrelated $=-0.5$ ), facial expression (effect coded as smiling $=+0.5$ and neutral $=-0.5)$ and the interaction between facial expression and relatedness. We included the rater ID and stimulus ID as random effects and specified our random slopes maximally (Barr, Levy, Scheepers, \& Tily, 2013). Analyses were conducted in the programming software $R$ version 3.5.0 ( $R$ Core Team, 2017) in conjunction with lme4 version 1.1.17 (Bates, Mächler, Bolker, \& Walker, 2015) and lmerTest version 3.0.1 (Kuznetsova, Brockhoff, \& Christensen, 2016).

As predicted, the analysis revealed a main effect of relatedness $(\beta=1.19, S E=0.19, z=6.09$, $p<.001$, odds ratio=3.29), whereby related pairs were 3.29 times more likely to be judged as related than unrelated pairs (see Figure 1). Both correct related judgments for related pairs $(\beta=0.48, S E=0.16, z=2.97, p=.003$, odds ratio=1.62) and correct unrelated judgments for unrelated pairs $(\beta=-0.71, S E=0.14, z=-5.22, p<.001$, odds ratio=0.49) were significantly above chance (not pre-registered hypotheses/analyses).

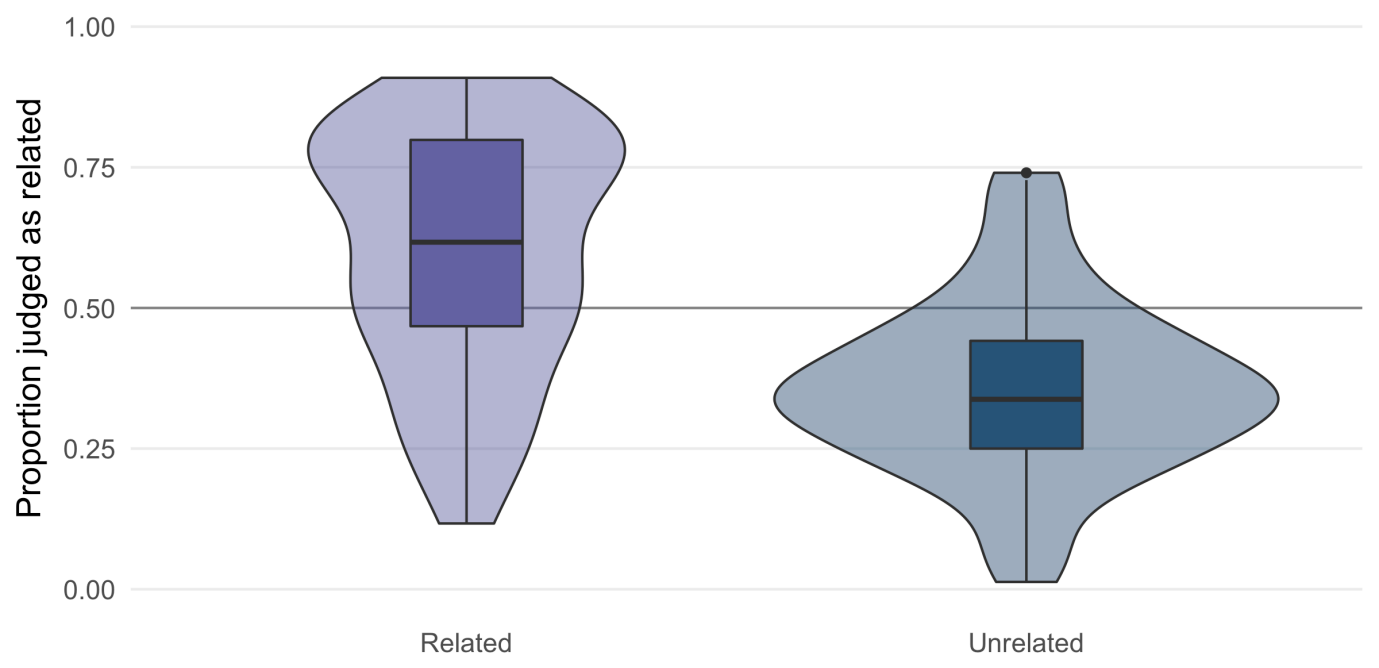

Figure 1: The main effect of relatedness on proportion of face pairs judged as related. 
There was no main effect of facial expression $(\beta=0.04, S E=0.08, z=0.56, p=0.573$, odds ratio $=1.04)$, but there was an interaction between facial expression and relatedness $(\beta=-0.42, S E=0.15, z=-2.69, p=0.007$, odds ratio $=0.66)$, whereby smiling related pairs were judged to be related less often than neutral pairs, while smiling unrelated pairs were judged to be related more often than neutral unrelated pairs (See Figure 2). This shows that a smiling facial expression decreases kinship judgment accuracy, contradicting our initial hypothesis.

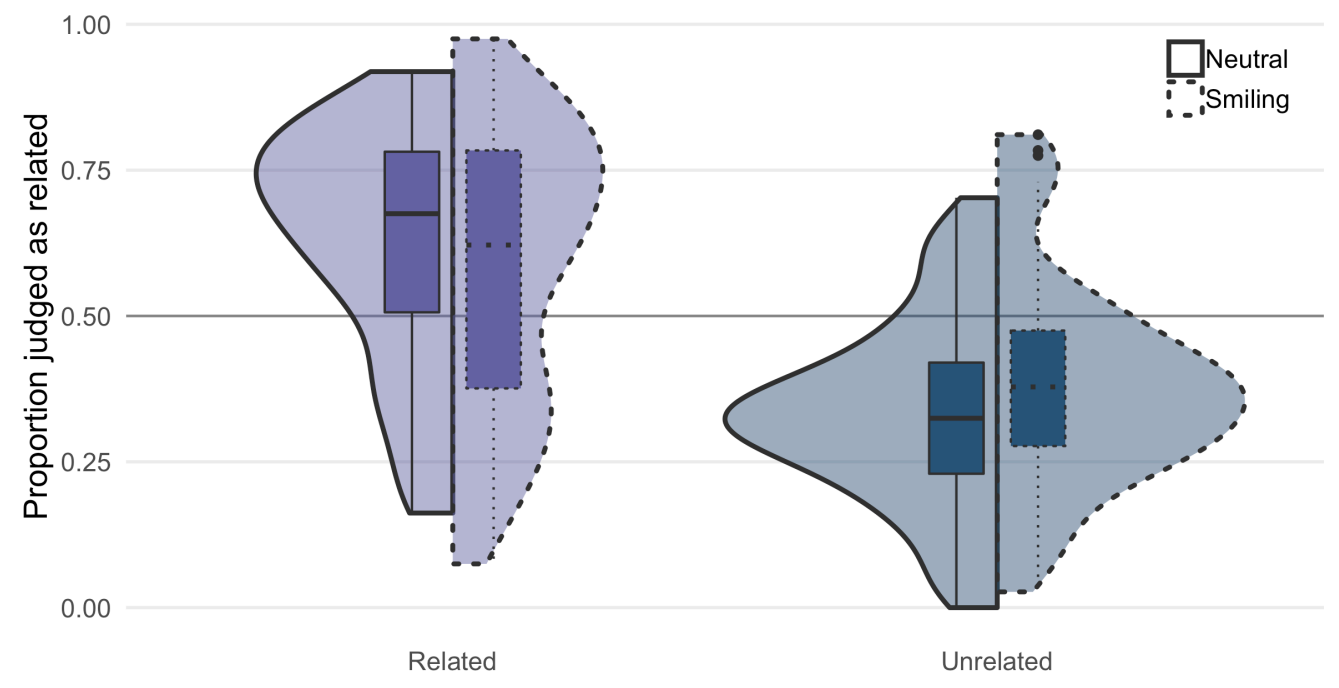

Figure 2: The interaction between relatedness and facial expression on proportion of face pairs judged as related.

We ran an exploratory (not pre-registered) analysis looking at possible effects of age gap within a pair on relatedness judgments. We repeated the analysis above, adding age gap as an additional factor. We found a significant interaction between age gap and relatedness $(\beta=-0.25, S E=0.11, z=-2.21, p=0.027$, odds ratio=0.78), whereby related pairs with a bigger age gap were less likely to be judged as related (See Figure 3). This analysis showed the same significant interaction between relatedness and expression as the pre-registered analysis $(\beta=-0.40, S E=0.15, z=-2.69, p=0.007$, odds ratio=0.67). 


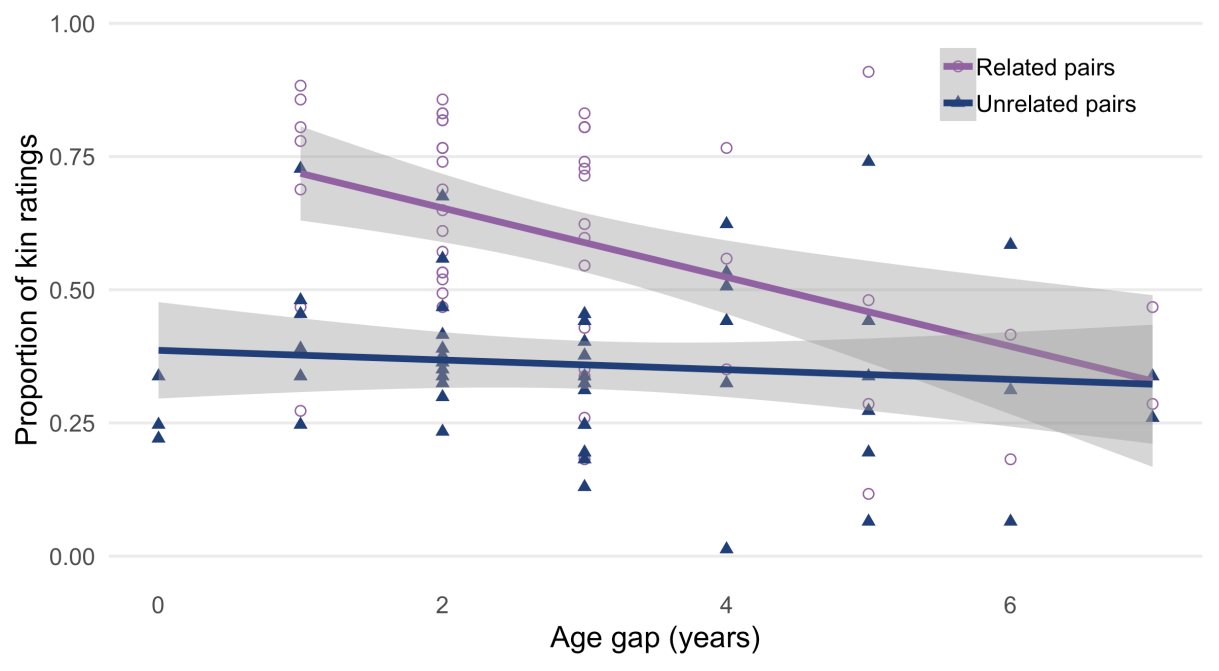

Figure 3: The interaction between relatedness and the age gap within a pair on proportion of face pairs judged as related.

\section{DISCUSSION}

In summary, we found that raters are able to discriminate siblings from unrelated pairs with some accuracy, which is consistent with previous literature (Alvergne et al., 2014; Bressan \& Dal Martello, 2002; Bressan \& Grassi, 2004; Dal Martello et al., 2015; DeBruine et al., 2009; Maloney \& Dal Martello, 2006). We also found that facial expression does influence kinship judgments, whereby a smiling facial expression decreases kinship judgment accuracy. In other words, related pairs were judged to be related less often when smiling than with a neutral expression, and unrelated pairs were judged to be related more often when smiling than with a neutral expression.

Marsh, Elfenbein and Ambady's $(2003,2007)$ found that a smiling facial expression aids the identification of an individual's nationality, yet, in the current study smiles do not aid the identification of kinship but hinder it. This could also suggest that smiles are partially a cue of common cultural background, but not a contextual cue of kinship, at least not in non-adult individuals, or that smiles as a possible cultural mannerism mask kinship cues.

One possible explanation for the finding that a smiling facial expression hinders kin recognition accuracy could be that conflicting mechanisms are employed when faces are processed for emotions and kinship. Previous research finds that the upper half of the face carries more kinship information than the lower half of the face (Dal Martello \& Maloney, 2006). However, when processing facial expressions of emotions observers focus on the mouth region (Schyns, Bonnar, \& Gosselin, 2002). Consequently, it is possible that an emotional expression could act as a distraction from processing facial cues that are informative of relatedness, as the mouth area is being attended to in order to process these expressions. Moreover, the lower part of the face undergoes radical shape and bone structure changes from childhood to adulthood (Kohn, 1991) and might therefore not be a reliable kinship cue, at least not in children. The current study used photographs of children who were between the ages of 3 and 17 years, hence a widely varying age group in terms of development of lower face characteristics. We found that a 
bigger age gap reduced the likelihood of related pairs to be judged as related. This could mean that kinship is harder to detect in related pairs when siblings are at different stages of facial development. The previously mentioned research finding that the upper half of the face carries more kinship information than the lower half of the face was also conducted with non-adult stimuli (Dal Martello \& Maloney, 2006). Consequently, we cannot readily conclude that facial expression in all cases decreases kinship judgment accuracy, as our results might be specific to kin with non-adult facial characteristics.

The current study provides further insight into our understanding and the nature of kinship detection. Our findings show that observers can identify sibling pairs and unrelated pairs at levels above chance, which is in line with previous research. Yet, a bigger age gap within related pairs reduced the likelihood of siblings being judged as related. Moreover, a smiling facial expression decreased the accuracy of judging relatedness compared to a neutral facial expression. This finding could be explained in light of previous research showing that the upper half of the face holds more information about relatedness than the lower half. The study would benefit from being replicated with adult stimuli, to account for facial changes due to growth.

\section{ACKNOWLEDGMENTS}

This research was supported by ERC grant \#647910 KINSHIP to L.M.D.

\section{REFERENCES}

Alvergne, A., Perreau, F., Mazur, A., Mueller, U., \& Raymond, M. (2014). Identification of visual paternity cues in humans. Biology Letters, 10(4), 20140063. DOI

Barr, D. J., Levy, R., Scheepers, C., \& Tily, H. J. (2013). Random effects structure for confirmatory hypothesis testing: Keep it maximal. Journal of Memory and Language, 68(3), 255-278. DOI

Bates, D., Mächler, M., Bolker, B., \& Walker, S. (2015). Fitting linear mixed-effects models using lme4. Journal of Statistical Software, 67(1), 1-48. DOI

Bateson, P. (1983). Mate Choice. Cambridge University Press.

Bressan, P., \& Dal Martello, M. F. (2002). Talis pater, talis filius: Perceived resemblance and the belief in genetic relatedness. Psychological Science, 13(3), 213-218. DOI

Bressan, P., \& Grassi, M. (2004). Parental resemblance in 1-year-olds and the gaussian curve. Evolution and Human Behavior, 25(3), 133-141. DOI

Butler, S., Gilchrist, I., Burt, D., Perrett, D., Jones, E., \& Harvey, M. (2005). Are the perceptual biases found in chimeric face processing reflected in eye-movement patterns? Neuropsychologia, 43(1), 52-59. DOI

Chapais, B., \& Berman, C. M. (2004). Kinship and behavior in primates. Oxford University Press on Demand.

Coolican, J., Eskes, G. A., McMullen, P. A., \& Lecky, E. (2008). Perceptual biases in processing facial identity and emotion. Brain and Cognition, 66(2), 176-187. DOI

Dal Martello, M. F., \& Maloney, L. T. (2006). Where are kin recognition signals in the human face? Journal of Vision, 6(12), 1356-1366. DOI

Dal Martello, M. F., \& Maloney, L. T. (2010). Lateralization of kin recognition signals in the human face. Journal of Vision, 10, 1-10. $\underline{\mathrm{DOI}}$ 
Dal Martello, M. F., DeBruine, L. M., \& Maloney, L. T. (2015). Allocentric kin recognition is not affected by facial inversion. Journal of Vision, 15(13), 1-11. DOI

DeBruine, L. M. (2002). Facial resemblance enhances trust. Proceedings of the Royal Society of London B: Biological Sciences, 269(1498), 1307-1312. DOI

DeBruine, L. M. (2004). Resemblance to self increases the appeal of child faces to both men and women. Evolution and Human Behavior, 25(3), 142-154. DOI

DeBruine, L. M., Jones, B. C., Little, A. C., \& Perrett, D. I. (2008). Social perception of facial resemblance in humans. Archives of Sexual Behavior, 37(1), 64-77. DOI

DeBruine, L. M., Smith, F. G., Jones, B. C., Roberts, S. C., Petrie, M., \& Spector, T. D. (2009). Kin recognition signals in adult faces. Vision Research, 49(1), 38-43. DOI

Dibeklioglu, H., Ali Salah, A., \& Gevers, T. (2013). Like father, like son: Facial expression dynamics for kinship verification. In Proceedings of the ieee international conference on computer vision (pp. 1497-1504). DOI

Hamilton, W. D. (1964). The genetical evolution of social behaviour. Journal of Theoretical Biology, 7(1), 1-16. DOI

Hepper, P. G. (2005). Kin recognition. Cambridge University Press. DOI

Kohn, L. (1991). The role of genetics in craniofacial morphology and growth. Annual Review of Anthropology, 20(1), 261-278. DOI

Krupp, D. B., DeBruine, L. M., \& Barclay, P. (2008). A cue of kinship promotes cooperation for the public good. Evolution and Human Behavior, 29(1), 49-55. DOI

Kuznetsova, A., Brockhoff, P. B., \& Christensen, R. H. B. (2016). LmerTest: Tests in linear mixed effects models. Retrieved from https://CRAN.R-project.org/package=lmerTest

Lewis, D. M. (2011). The sibling uncertainty hypothesis: Facial resemblance as a sibling recognition cue. Personality and Individual Differences, 51 (8), 969-974. DOI

Lieberman, D., Tooby, J., \& Cosmides, L. (2007). The architecture of human kin detection. Nature, 445(7129), 727. DOI

Maloney, L. T., \& Dal Martello, M. F. (2006). Kin recognition and the perceived facial similarity of children. Journal of Vision, 6, 1047-1056. DOI

Marsh, A. A., Elfenbein, H. A., \& Ambady, N. (2003). Nonverbal "accents" cultural differences in facial expressions of emotion. Psychological Science, 14(4), 373-376. DOI

Marsh, A. A., Elfenbein, H. A., \& Ambady, N. (2007). Separated by a common language: Nonverbal accents and cultural stereotypes about americans and australians. Journal of Cross-Cultural Psychology, 38(3), 284-301. DOI

Megreya, A. M., \& Havard, C. (2011). Left face matching bias: Right hemisphere dominance or scanning habits? Laterality, 16(1), 75-92. DOI

Nesse, R. M., Silverman, A., \& Bortz, A. (1990). Sex differences in ability to recognize family resemblance. Ethology and Sociobiology, 11(1), 11-21. DOI

Penn, D. J., \& Frommen, J. G. (2010). Kin recognition: An overview of conceptual issues, mechanisms and evolutionary theory. Animal behaviour: Evolution and mechanisms (pp. 55-85). Springer. DOI

Platek, S. M., Critton, S. R., Burch, R. L., Frederick, D. A., Myers, T. E., \& Gallup Jr, G. G. (2003). How much paternal resemblance is enough? Sex differences in hypothetical investment decisions but not in the detection of resemblance. Evolution and Human Behavior, 24(2), 81-87. DOI

R Core Team. (2017). R: A language and environment for statistical computing. Vienna, Austria: $\mathrm{R}$ Foundation for Statistical Computing. Retrieved from https://www.R-project.org/

Rhodes, G. (1985). Lateralized processes in face recognition. British Journal of Psychology, 76(2), 249-271. DOI 